\title{
The Meta-analytic Saga of Serotonin Reuptake Inhibitors in an Obsessional World
}

\section{BY STEVEN A. RASMUSSEN, MD}

It was only 20 years ago that obsessivecompulsive disorder (OCD) was thought to be a rare illness with a poor prognosis. In contrast to their colleagues of 20 years ago, clinicians now have the luxury of choosing between five potent inhibitors of serotonin reuptake, including clomipramine, fluvoxamine, fluoxetine, sertraline, and paroxetine, all of which have proven efficacious in reducing obsessive-compulsive symptoms when compared with placebo in multicenter doubleblind placebo-controlled trials. In addition, the development of new behavioral treatment strategies for patients with pure obsessions or cognitive compulsions, coupled with increasing evidence of the long-term efficacy of behavior therapy in addition to its ability to prevent relapse following SRI withdrawal in maintenance treatment, has added significantly to the clinician's armamentarium in treating this chronic and frequently disabling condition.

Randomized double-blind trials that have directly compared the efficacy and safety of one SRI with another in the treatment of OCD have in general confirmed clinical impressions that the drugs are roughly equivalent in acute efficacy, with the selective serotonin reuptake inhibitors being better tolerated than the tricyclic clomipramine but perhaps less efficacious. The early clinical impression that clomipramine may be somewhat superior in efficacy to the selective serotonin reuptake inhibitors (SSRIs) has not been confirmed in these trials.

However, the fact that there were relatively few subjects in these studies makes the likelihood of a type II error high. A meta-analytic study by Greist et al ${ }^{1}$ of all the doubleblind multicenter controlled trials suggested that clomipramine was superior in efficacy to the SSRIs. There are many methodologic issues in such a study that make firm conclusions difficult. Perhaps the most significant of these issues was the fact that almost all of the subjects in the initial clomipramine trial were naive to pharmacologic intervention. In contrast, $40 \%$ of the subjects in the sertraline trial (the last to be undertaken in the US) had failed a previous trial of an SRI (Rasmussen, unpublished data). A recent reanalysis of that data suggests that there was no significant difference in the effect size when patients who had been treated with an SRI previously were excluded from the analysis. There are data to support the strategy of switching to a second SRI if the first has failed. Analysis of the multicenter fluvoxamine trial has shown that $20 \%$ of patients who failed to respond to a previous trial of either clomipramine or fluoxetine went on to respond to a 10 week trial of fluvoxamine. ${ }^{2}$

Differences in the side effect profiles and pharmacokinetics of the various SRIs can also influence choice of drug. Many patients have difficulty tolerating the acute anticholinergic and antiadrenergic side effects of clomipramine. The prolonged half-life of fluoxetine confers an advantage when one is dealing with a potentially noncompliant subject but is problematic when it is important to discontinue the drug rapidly, as in when the patient has bipolar manic tendencies or becomes pregnant.

Another important consideration is comorbid symptoms. For example, if patients have considerable apathy and fatigue, an activating compound such as fluoxetine might be considered. In contrast, for patients with high levels of agitation or panic, one might wish to consider a less activating compound. A great deal has been written about differences in P-450 enzyme systems between the SRIs. While it is important to be aware of the contraindications for concomitant drug administration with the SRIs, in practice, it is rare that this is a determining factor in drug selection. Finally, it is important to recognize that although a majority of patients are significantly improved with the SRIs, 30\% are treatment refractory, and of those that do significantly improve, most are left with residual symptoms. For these patients, adding behavior therapy is an important therapeutic consideration. CVS

Dr. Rasmussen is medical director at Butler Hospital, Providence, $R I$, and associate professor of psychiatry and human behavior at Brown University, Providence, RI.
"Randomized double-

blind trials that have

directly compared the

efficacy and safety of

one SRI with another

in the treatment of

OCD have in general

confirmed clinical

impressions that the

drugs are roughly

equivalent in acute

efficacy, with the

selective serotonin

reuptake inhibitors

being better tolerated

than [tricyclics] but

perhaps less

efficacious."

\section{REFERENCES}

1. Greist JH, Jefferson JW, Kobak $\mathrm{KA}$, et al. Efficacy and tolerability of serotonin transport inhibition in obsessive-compulsive disorder: a metaanalysis. Arch Gen Psychiatry. 1995; 52:53-60.

2. Rasmussen SA, Goodman WK, Greist JH, et al. Fluvoxamine in the treatment of obsessivecompulsive disorder: a multicenter, double-blind, placebocontrolled study in outpatients. $J$ Clin Psychopharmacology. Submitted, 1996. 